\title{
Fatigue design of welded joints using the finite element method and the 2007 ASME Div. 2 Master curve
}

\author{
T. Marin, G. Nicoletto \\ University of Parma, Department of Industrial Engineering, Italy, marin@ied.unipr.it
}

RiassunTO. Lo studio della fatica nei giunti saldati è solitamente basata sugli approcci della tensione nominale, quella di hot spot o quella di intaglio, i quali però presentano svariate limitazioni quando sono associati ad analisi mediante il metodo degli elementi finiti. In questo lavoro viene presentata una definizione più recente di tensione strutturale e la sua implementazione in un postprocessore per codici FEM. Essa permette un efficace utilizzo dei risultati delle simulazioni per valutare la resistenza a fatica di strutture anche complesse. Le applicazioni presentate confermano le principali caratteristiche del metodo, ossia l'insensibilità alla mesh e stime accurate della vita a fatica e del punto di propagazione delle cricche.

\begin{abstract}
Fatigue design of welded structures is primarily based on a nominal stress; hot spot stress methods or local approaches each having several limitations when coupled with finite element modeling. An alternative recent structural stress definition is discussed and implemented in a post-processor. It provides an effective means for the direct coupling of finite element results to the fatigue assessment of welded joints in complex structures. The applications presented in this work confirm the main features of the method: mesh-insensitivity, accurate crack location and life to failure predictions.
\end{abstract}

KEYWORDS. Welded joints; Structural stress; Fatigue assessment; Finite element analysis.

\section{INTRODUCTION}

$\mathrm{W}$

elding is the most widespread joining technique for metallic structures due to its applicability to many geometric configurations. The principal failure mode in welds is fatigue cracking, [1], therefore many efforts have been put in the study of the strength of welded joints and in the definition of design guidelines, [2]. As the finite element method has become the favorite tool in structural analysis, there is an unquestionable need for a direct connection of the fatigue assessment approaches to the simulations.

In this work, after a brief review of some well established techniques in Section 2, a novel approach developed at Battelle Institute, and recently entered in the ASME standards, is described in Sections 3-4. Some applications to experimental tests are then presented in Section 5. This method allows a relaxation of some finite element modeling difficulties, mainly the mesh sensitivity, and grants the possibility of using alternatively solid and shell elements to model welded joints. The results of the $\mathrm{FE}$ analysis are then used in combination to a fatigue master curve that consolidates a large number of welded joint configurations.

\section{APPROACHES TO FATIGUE ASSESSMENT OF WELDED JOINTS}

$\mathrm{I}$

$\mathrm{t}$ is common practice to separate the approaches to fatigue assessment of welded joints into "global" and "local" families, [3-4]. The former consists of the long standing nominal stress method, which is still the most used for its simplicity and is the basis of all standards and design codes in use. With nominal stress approach no attempt is made 
of taking into account the stress concentrations due to macro- and micro-geometric effects of the joints. It employs several empirical S-N curves that are associated with detail categories and corrective factors. The selection of a detail class for a welded joint type and loading mode is often subjective and, in many common situations, difficult even for a skilled engineer. This is especially true when the geometry of the structure is complex or when the stress state is not reducible to a simple main component. Moreover it must be added that the real structures can develop cracks in locations different to those indicated in the details present in the standards so this method has severe limitations.

The group of "local" methods comprises many different strategies, ranging from the notch stress and notch strain approaches to the fracture mechanics approach. A brief generalization of them is not possible since they differ in the local parameter (being a stress, a strain or a stress intensity factor) and in the phase of the fatigue damage where they can be applied (for example local notch stress is suitable for the crack initiation while fracture mechanics is ideal for crack propagation), [5]. Even if these approaches are sophisticated and have a significant theoretical foundation, the applicability is very often confined to specific cases and therefore they cannot be easily generalized to cover the variety of situations typically found in engineering. This is the main reason why they have not seen a straightforward acceptance in the standard codes, [3].

An intermediate approach between "global" and "local" methods uses a definition of a representative stress, in proximity of the weld toes, which is based on an idealized stress distribution in the thickness of the joined members. Different terms have been adopted for this stress depending on the field of application and on the way it is calculated (geometric stress, structural stress, hot-spot stress). Here the term structural stress is adopted. The fundamental idea is to consider the stress component orthogonal to the weld line and to reduce it to a linearized distribution, Fig. 1a. The structural stress approach is suited for the assessment of fatigue failures occurring at the weld toes; accordingly it is the stress component normal to the crack plane, i.e. normal to the weld line that is the driver for crack propagation (Fig. 1b).
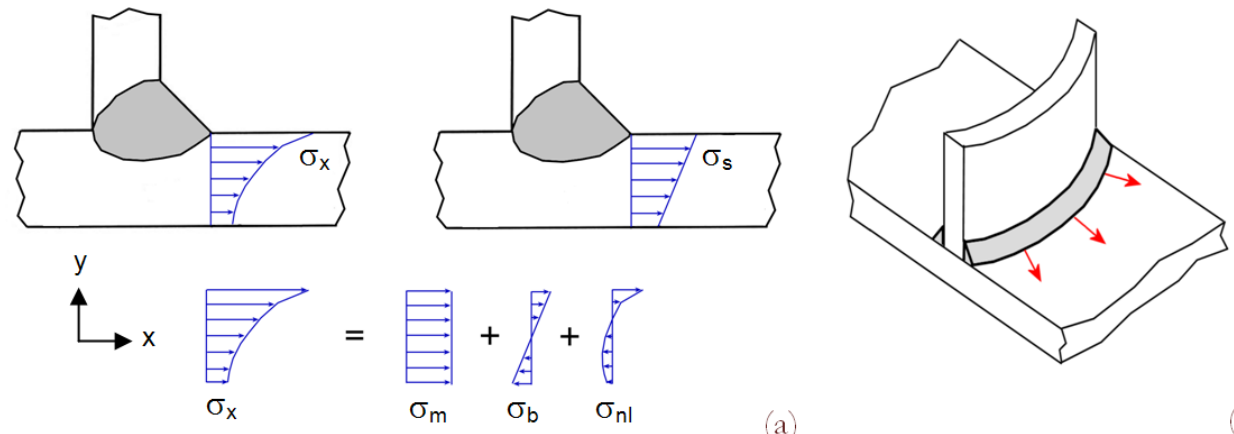

(a)

(b)

Figure 1: a) Decomposition of the through thickness stress at the weld toe; b) stress component acting normal to the weld fillet.

The structural stress can be inferred by surface measurements and extrapolations, leading to the traditional hot-spot technique. The procedure can be replicated by numerical simulations using finite element models and is present in standard codes (i.e. Eurocode3). Linearization of the stress over the section thickness can be achieved only through FE simulations and usually interrogating nodal stresses. Such practiced has also been introduced in pressure vessel standard EN 13445. As a result of the linearization, the structural stress $\sigma_{\mathrm{s}}$ at the weld toe is composed by a membrane part $\sigma_{m}$, constant in the thickness, and a bending part $\sigma_{b}$, as depicted in Figure 1a. The remaining self-equilibrated non-linear $\sigma_{n l}$ is not considered; therefore the structural stress includes only the effects of gross structural discontinuities but disregards the local notch effect due to the weld geometry. The notch-induced complex stress state at the weld toe can then be simplified and only the two components $\sigma_{m}$ and $\sigma_{b}$ are taken into account. The finite element simulations required for this approach are linear elastic and the fatigue assessment is performed using structural stress $\mathrm{S}-\mathrm{N}$ curves that are in limited number with respect to the nominal stress S-N curves.

\section{STRUCTURAL STRESS APPROACH BASED ON NODAL FORCES}

$\mathrm{T}$ he finite element framework allows the calculation of a structural stress based on forces and moments at the nodes of the mesh. This method has the distinctive advantage of providing a structural stress fairly insensitive to the mesh features (element size and element type) in the areas corresponding to the weld toes. The typical meshdependence that is found in the traditional surface extrapolation method and in the through thickness linearization, is 
therefore overcome. Elemental stresses or stresses extrapolated to the nodes are in fact influenced by the element formulation and by the geometric characteristics of the finite elements, whereas nodal forces directly derive from the equilibrium of the structure. Nodal forces (and moments) for each element are calculated from the stiffness matrix and the nodal displacements (and rotations). The displacements are the primary output of displacement-based FE codes and the equilibrium at each node in the mesh is satisfied regardless of the element size and element formulation. A few different variants of this approach have been proposed and they mainly stem from the automotive field for either spot or seam welds, see for example Fermer and co-workers [6]. The literature reports also a recent implementation of a similar approach in the commercial code Femfat, [7]. Researchers, headed by P. Dong at Battelle Institute, have formulated an effective procedure for the calculation of the structural stress from forces and moments at the nodes of a finite element mesh, based on work-equivalence considerations, [8]. In Dong's method, first distributed line forces (and moments) are determined along the edges of the weld toe lines starting from balanced nodal forces (and moments), then at each node the structural stress is calculated as:

$$
\sigma_{s}=\sigma_{m}+\sigma_{b}=\frac{f_{y}}{t}+\frac{6 \cdot m_{x}}{t^{2}}
$$

where $t$ is the section thickness of the plate, $f_{y}$ is the line force in the local $y$ direction orthogonal to the weld line and in the plane of the shell; $m_{x}$ is the line moment in the local $x$ direction tangent to the weld line. Forces and moments have to be preliminarily rotated into local coordinate systems defined at the nodes of the weld line. The resulting line forces (and moments) are continuous along the weld toe lines and so is the structural stress. The detailed procedure is described in several publications, for example [8-9].

Even if these concepts can be applied to solid elements (2D and 3D), the approach is particularly suited for shell elements. Thus, since shell and plates are often the preferred choice for modeling the response of engineering structures that are obtained using welding (for example truck frames, ships, cranes, bridges, etc.), the potential applications of the method are many. It must be emphasized that the finite element simulations have to be linear elastic therefore a fatigue assessment of the welded joints in the components can be a precious additional outcome of a standard stress analysis. The only specific requirement concerns the modeling of the welds because the fillets must be explicitly included to correctly represent the stiffness of the joints. This can be done using inclined elements, as shown in Fig. 2a which reports an example of a T-joint connection between two tubular parts. Several strategies for a realistic modeling of partial and complete penetration seam welds are collected in Fig. 2b. The authors acknowledge that the manual creation of the elements in the welds is a tedious and time consuming part of the procedure and the automation of the welds definition has to be pursued.

Note in Fig. 2a how for a given fillet both the two toe lines have to be analyzed since a priori it is not know which one is the most prone to fatigue propagation and where.
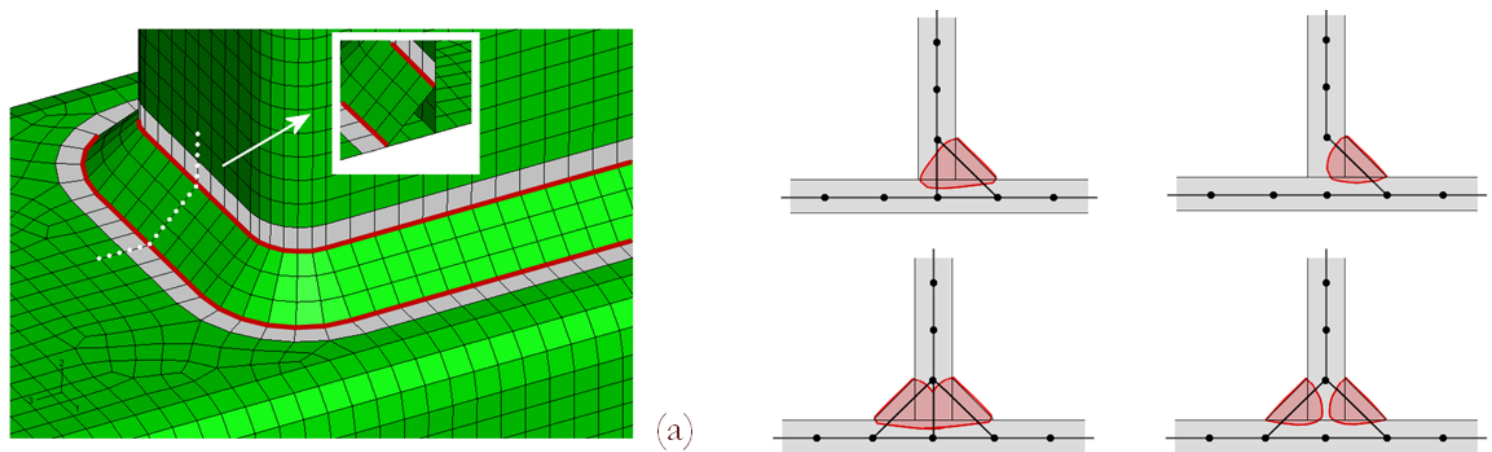

(a)


(b)

Figure 2: a) Tubular connection (T-joint) modeled with shell elements; b) fillet welds with partial and complete penetration.

The mesh-insensitivity of the structural stress claimed above, is demonstrated through the illustrative numerical example of Figure 3. Here a curved thin profile is joined on the outer side to a flat plate by a full penetration fillet weld. The main dimensions of the flat panel are $100 \times 100 \mathrm{~mm}$, the two sides of the curved plate are $50 \mathrm{~mm}$ long and the thickness is 5 $\mathrm{mm}$. A uniform traction is applied longitudinally to the curved profile and transverse loading acts on the top edge as shown in Fig. 3a; top and bottom nodes of the flat plate are pinned. Three different meshes are studied, the first one is shown in Fig. 3a together with the resulting V.Mises stress on the visible surfaces of the shells. This is a rather coarse 
mesh but regularly spaced and with a low aspect ratio in the rows of elements corresponding to the two weld toes. The second case considered, Fig. 3b, has weld toes characterized by distorted elements having a wide spectrum of shape metrics. Fig. 3c shows the third mesh with a regular and fine discretization. Any experienced finite element analyst would prefer the last one for a local stress investigation but such level of refinement may not be necessary for a fatigue analysis based on the present procedure. The proof comes from Figure 4 where the structural stress $\left(\sigma_{s}\right)$, the membrane $\left(\sigma_{m}\right)$ and bending $\left(\sigma_{b}\right)$ components are plotted along the weld toe lines 1 and 2 indicated in Fig. 3a. The results from the three meshes are drawn with different line styles (refined mesh: continuous lines, distorted mesh: dashed lines, coarse mesh: dash-dot lines) but they are barely distinguishable since they are closely overlapped. The distorted mesh plots have few little jerks in correspondence of some elements but the trends are completely captured and so are the peak values and positions. Even the coarse mesh does not fail in revealing the maximum values. These graphs give also useful information about the relative magnitude of the structural stress components, membrane and bending, so the analyst has a clear picture of the loading mode in each point along the weld toes. In the example discussed here there is a strong predominance of bending stress in most parts of the toe lines.
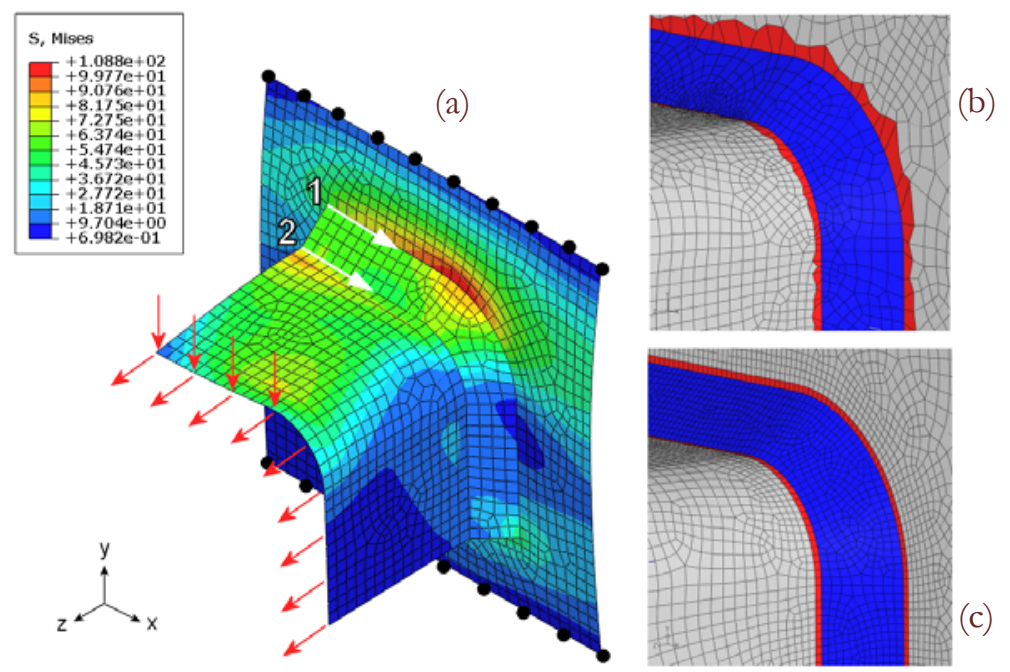

Figure 3: Curved profile welded to a flat plate: a) shell model using a coarse regular mesh at the toes; b)-c) close views of the fillet for the irregular mesh and the refined regular mesh.


Figure 4: a) Comparison of the structural stress and its membrane and bending components along the weld toes of Fig. 3. FM: fine mesh mesh, CM: coarse mesh, DM: distorted mesh. 
The mesh sensitivity of the nodal stresses extrapolated to the nodes on the toe line is obvious in Fig. 5. These are the normal stresses to the weld, calculated as weighted average of the stresses (extrapolated to the nodes) given only by the elements in the toe. The convergence of the mesh is evident since the coarse mesh provides lower stresses but, even if the finer mesh provides higher stresses, these values are still far from the structural stress calculated based on nodal forces.

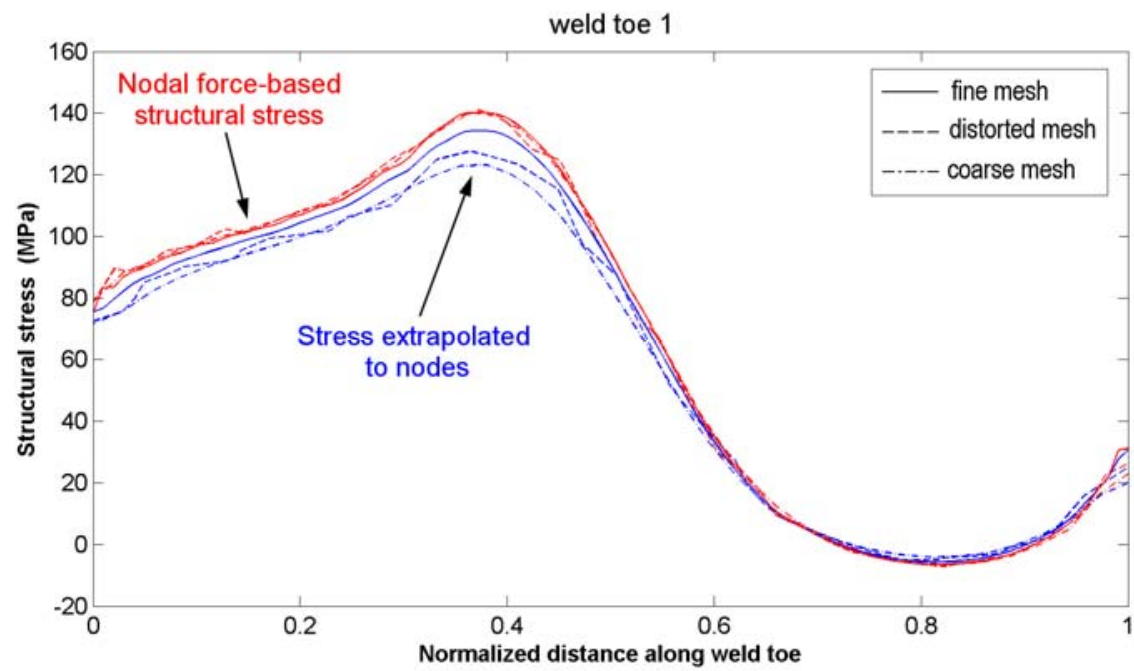

Figure 5: Comparison of the normal stress to the weld calculated from nodal stresses and the structural stress obtained from nodal forces (toe 1 in Fig. 3).

One of the major drawbacks of the structural stress approaches in their basic forms is that they usually take into account only the stress component normal to the weld line. In analogy with Eq. (1), a structural shear stress along the fillet could be calculated as:

$$
\tau_{s}=\tau_{m}+\tau_{b}=\frac{f_{x}}{t}+\frac{6 \cdot m_{y}}{t^{2}}
$$

As of now only few studies on multiaxial fatigue and structural stress are found in the literature, [10], so a complete understanding of the correct combination of $\sigma_{s}$ and $\tau_{s}$ is yet to be available.

The whole procedure has been implemented by the authors in Matlab routines which act as a post-processor to the FE software Abaqus. Together with the calculations described above, the code detects the toes for all the fillets in the shell mesh of a structure or a component, thus offering an automatic, quick and complete analysis of the welded joints.

\section{THE ASME MASTER S-N CURVE}

A ccording to this approach, the structural stress defined in Equation 1 is consistent with the far-field stress typically used in fracture mechanics to compute the stress intensity factors $K$ for a given crack shape and size. Since the life of welded joints is dominated by crack propagation, the structural stress and its components correlate the actual geometry and loading of any joint to simplified fracture mechanics configurations where crack growth models can be applied. As a result of the analytical procedure developed in [9] for a two-stage growth model, an equivalent structural stress parameter can be defined as:

$$
\Delta S_{s}=\frac{\Delta \sigma_{s}}{t^{(2-m) / 2 m} \cdot I(r)^{1 / m}}
$$

where the structural stress range $\Delta \sigma_{s}$ is modified by a loading mode function $I(r)$ and by a thickness correction factor. The polynomial $I(r)^{1 / m}$ is a function of the ratio $r$ : 


$$
r=\frac{\Delta \sigma_{b}}{\Delta \sigma_{m}+\Delta \sigma_{b}}
$$

that represents the content of bending stress over the total structural stress, $t$ is the section thickness of the plate and $m=3.6$ represents the slope of a Paris-like crack propagation curve.

It has been shown in several publications by Dong and co-workers that, using this procedure, it is possible to define a single S-N curve for many different weld geometries and loading configurations, therefore proving its robustness. This master S-N curve, which has also been incorporated in the ASME Boiler and Pressure Vessel Codes (2007) Section VIII Division 2 as an alternative prediction method, has the following form:

$$
\Delta S_{s}=C \cdot N^{h}
$$

where $C$ and $h$ are parameters of the material and are tabulated for different prediction intervals. Contrary to most of the standards, this norm does provide neither a cut-off limit (fatigue strength for infinite life) nor any knees in the curve: all the cycles (after rainflow filtering) are considered damaging. This is consistent with the recommendations given in [11].

The equivalent structural stress is the parameter that provides the estimate of the life via Eq. (5). These concepts can again be applied to the example from Section 3. Accepting that the combined loading is proportional and in-phase, and assuming that the load history is constant amplitude with a stress ratio $R=0$, the structural stress range is $\Delta \sigma_{s}=\sigma_{s}$. The number of cycles to failure is then obtained through Eq. (3)-(5) and the results are given in the graphs of Figure 6. The point with the maximum $\Delta S_{s}$ is the location where the fatigue cracks would most probably propagate in the through thickness of the plate. In this case the plots suggest that a fatigue crack would take place at $0.4^{*} L$ of the toe 1 ( $L$ : total length of the toe line) and that the failure of the part would occur after about $4.0 \mathrm{E}+6$ cycles based on the mean master S$\mathrm{N}$ curve.
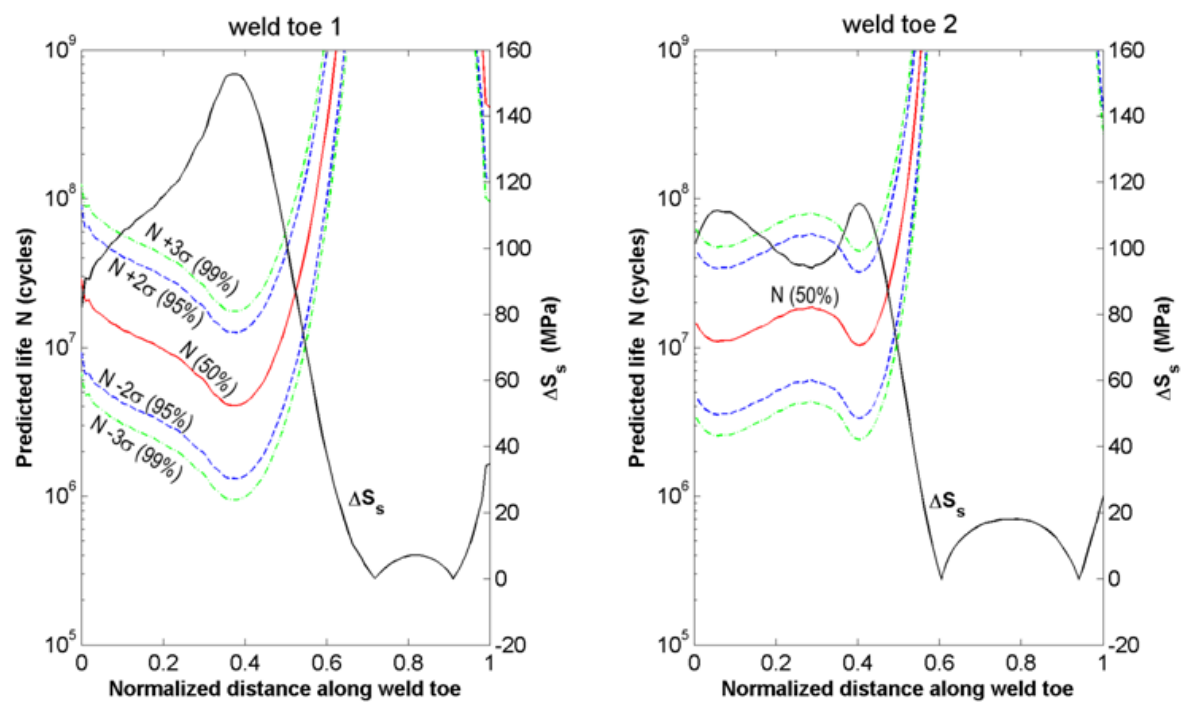

Figure 6: Life prediction (with different scatter bands) and equivalent structural stress range $\Delta \mathrm{S}_{\mathrm{s}}$ for the weld toes in Fig. 3.

\section{Applications}

$\mathrm{S}$ ome experimental tests were performed in this work to further validate the structural stress approach and ASME master S-N curve. Several specimens of three different geometries were subjected to pulsating tensile constant amplitude loading (stress ratio $R=0$ ). To assess the predictive capabilities of the method, the maximum load was set to values corresponding to a given number of cycles. From the target life, the structural stress was deduced using Eq. (3)(5) and compared to the maximum structural stress found in a finite element simulation of the specimen subjected to the known load. The linearity of the solution then allowed an easy scaling of the applied load to determine the force required in the test. The target life of this experimental campaign ranged from $\mathrm{N}=1.0 \mathrm{E}+5$ to $\mathrm{N}=5.0 \mathrm{E}+5$. 
The specimens consisted of plates (thickness $t=8$ or $10 \mathrm{~mm}$, width $w=50 \mathrm{~mm}$, length $L$ ranging from 250 to $300 \mathrm{~mm}$ ) with attachments on the top surface. The attachments were H-shaped, T-shaped and a solid cubic block ("H", "T", "B" types in the following). The specimen types " $\mathrm{H}$ " and " $\mathrm{T}$ " had $5 \mathrm{~mm}$ thick attachments that were centered and aligned with the longitudinal direction, while the solid block $(37 \times 37 \times 30 \mathrm{~mm})$ was not symmetrically positioned on the top of the plate. The material was a typical structural steel Fe510. The failure criterion adopted was the complete propagation of the fatigue cracks through the plate thickness, so the tests were continued until final fracture had occurred and the total number of cycles could be determined.

A detailed presentation of the computed structural stress is provided for type "T" specimen. Fig. 7 shows two finite element models constructed using shell elements. Because of the particular geometry, the T-shaped attachment is completely wrapped by the inclined elements forming the fillet. The simplified toe line in Fig. 7b is continuous and smooth except in two points where there is an abrupt change in direction and a $90^{\circ}$ angle. At these points (marked with a $\mathrm{P}$ in the figure) some disturbance in the structural stress distribution has to be expected.
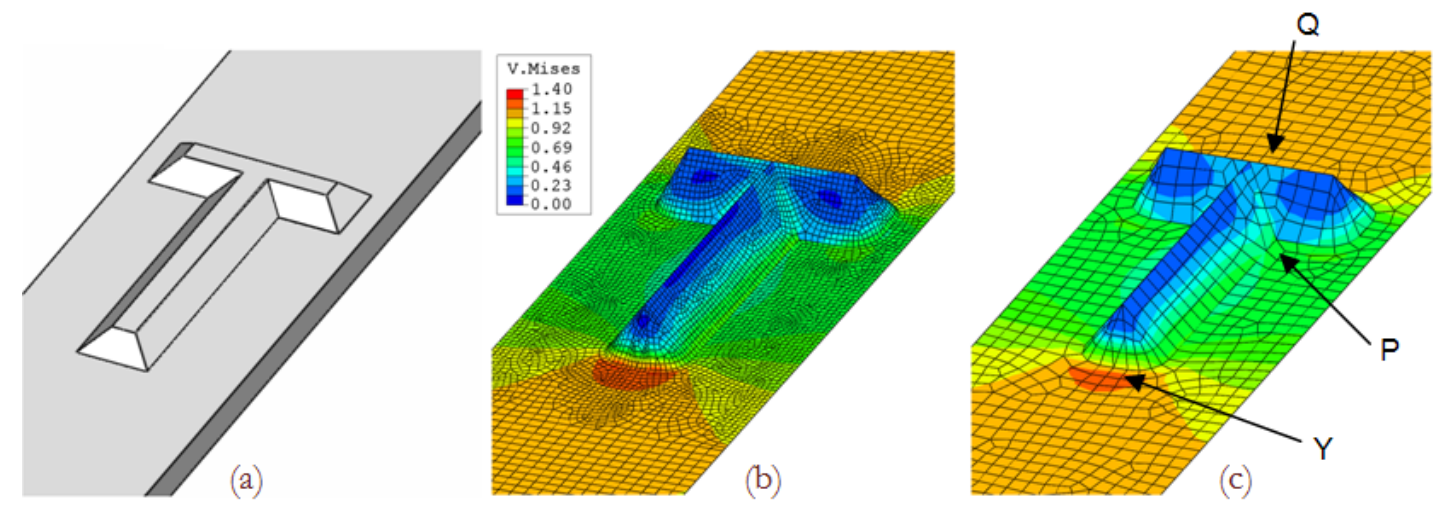

Figure 7: a) Specimen with the T-shaped attachment; b)-c) shell models with different mesh size. The V.Mises stress is normalized by the nominal tensile stress.

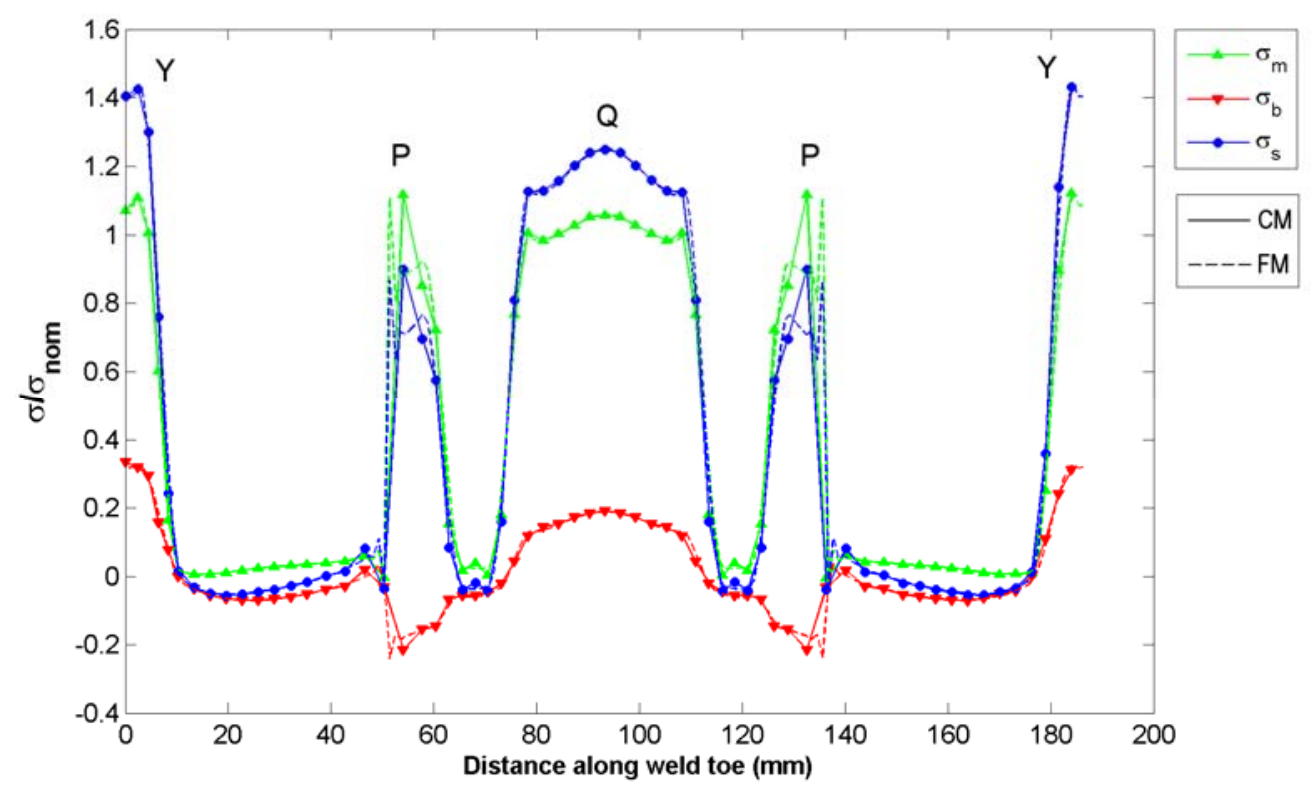

Figure 8: Structural stress and its components along the fillet weld of type "T" specimen. CM: coarse mesh, FM: fine mesh.

The results of the post-processed simulations are illustrated in Fig. 8 for two mesh sizes. The graph has in the x-axis the position of the nodes on the toe line with respect to a curvilinear abscissa with origin on the tip of the " $T$ " and running along the whole fillet. The continuous lines refer to the coarse mesh while the dashed lines correspond to the fine mesh. The structural stress values are normalized by the nominal stress in the section $\left(\sigma_{n o m}=F / A, F\right.$ : applied force, $A$ : area of 
the transverse section). These stresses are to be intended as located on the upper surface of the plate and at the intersection of the weld elements and plate elements. The graph shows that there is an obvious symmetry due to the geometry, and, above all, that the fine mesh and coarse mesh plots are consistently overlapped with differences substantially negligible almost everywhere. There are some sensible discrepancies only close to the points $\mathrm{P}$ as discussed before. It is evident from the graph that the membrane content is dominant as could have been expected since the specimen is loaded in tension. The macro-geometric notch effect caused by the attachment is responsible for the bending component. Peak values are reached at the tip of the "T" (point Y in Fig. 8) and in the middle point of the top leg of the "T" (point Q in Fig. 8). These are actually the locations of the fatigue failures, the choice between Y and Q being dictated by the local weld quality. Along the sides of the "T", which are parallel to the load direction, the structural stress is close to zero because only the stress component normal to the weld fillet is considered.
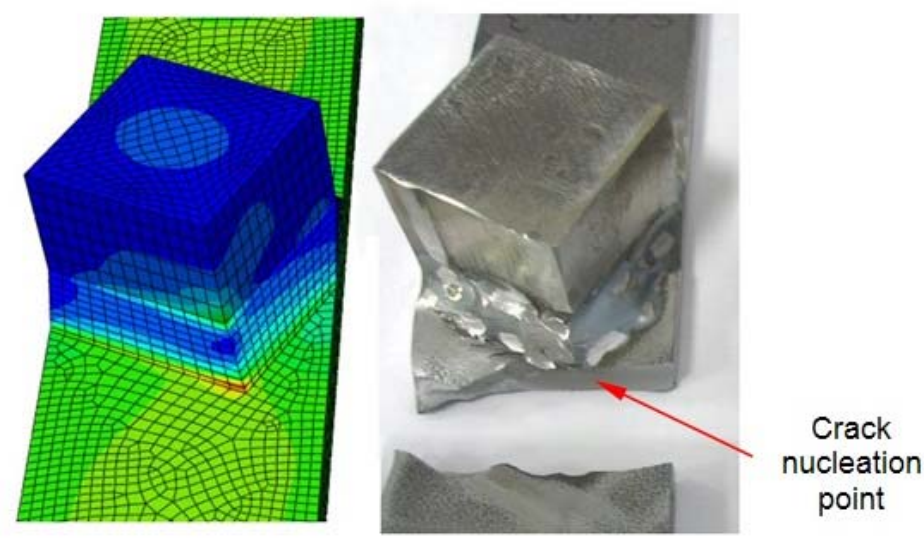

Figure 9: Finite element model using 3D elements and real specimen with the solid block.

Analogous plots are obtained for type " $\mathrm{H}$ " specimens and for sake of brevity are not reported. The presence of the massive block in type "B" specimens prevents the use of shells so for that case solid 3D elements are employed, Fig. 9. It is important to note that the failure location is always in correspondence with the highest value of the structural stress so these tests confirm the ability of the method to take into account the main features of fatigue failure in a simplified but correct way. The fatigue data for the all the specimen types are inserted into the 2007 ASME Div. 2 Master curve as shown in Fig. 10. All the data, independently of the specimen geometry, fall within the $\pm 2 \sigma$ S-N curves, therefore demonstrating that the predictions using the presented calculation approach are quite accurate.

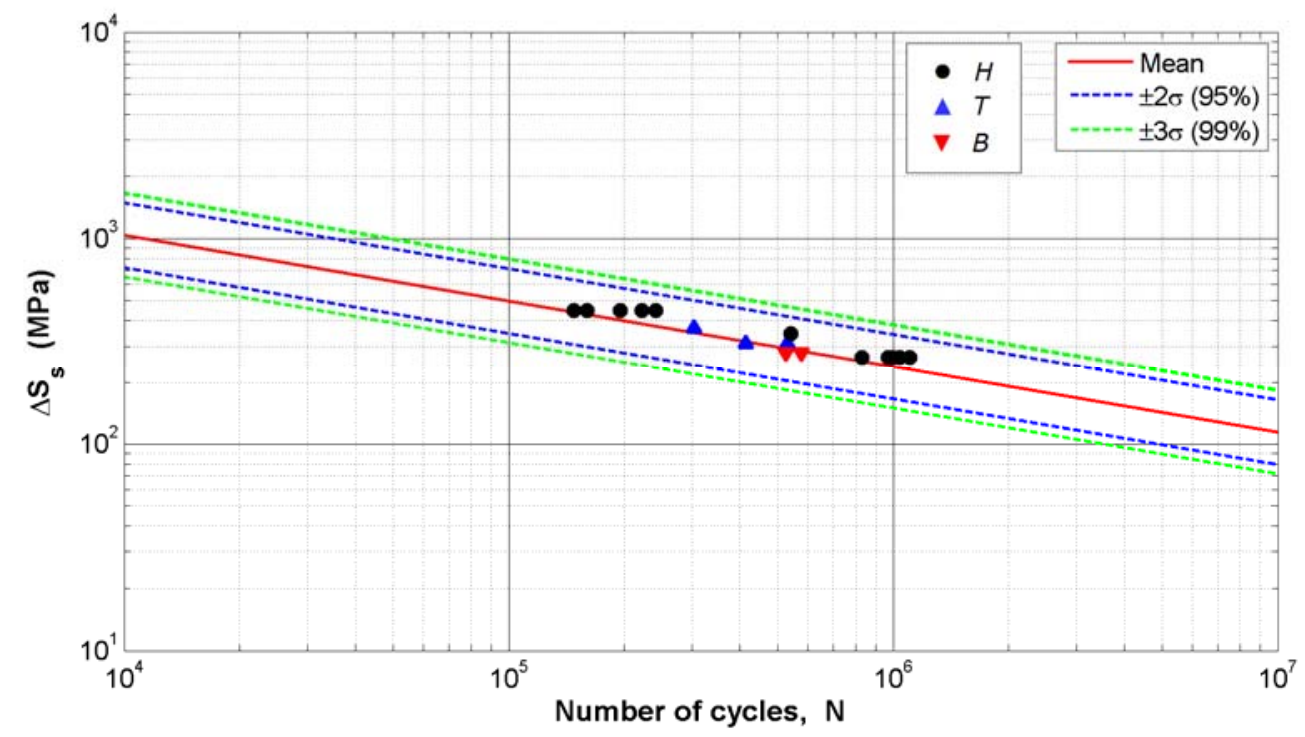

Figure 10: 2007 ASME Div.2 master S-N curve and experimental results. 


\section{CoNCLUSIONS}

he work presented a structural stress approach to fatigue assessment of welded joints that integrates well with finite element modeling. The implementation in a post-processor program was successful and showed the potential for becoming a useful tool for the design and assessment of welded structures subjected to fatigue.

The mesh-insensitivity was confirmed: even coarse meshes provide adequate structural stress estimates so the method can be used in modeling complex structures.

The procedure was applied to three different specimen geometries subjected to constant amplitude loading and predicted the correct location of the fatigue cracks. Finally, the use of the ASME master S-N curve proved to give accurate fatigue life predictions.

\section{REFERENCES}

[1] D. Radaj Design and Analysis of Fatigue Resistant Welded Structures, Abington Publishing, Cambridge (1990).

[2] W. Fricke, Marine Structures, 16 (2003) 185.

[3] D. Radaj, C.M. Sonsino, W. Fricke Fatigue Assessment of Welded Joints by Local Approaches (2nd Ed.), Woodhead Publishing, Cambridge (2006).

[4] A. Hobbacher, Recommendations of IIW Joint Working Group XIII - XV, Woodhead Publishing (1996).

[5] B. Atzori, P. Lazzarin, R. Tovo, Fatigue and Fracture of Engineering Materials and Structures, 22 (1999) 369.

[6] M. Fermer, M. Andreasson, SAE Technical Paper 982311(1998).

[7] S. Zhang, DaimlerChrysler AG Research Report GR/VMB-07-001 (2007).

[8] P. Dong, Int. J. of Fatigue, 23 (2001) 865.

[9] P. Dong, J. K. Hong, Z. Cao, Int. J. of Fatigue, 25 (2003) 811.

[10] P. Dong, J. K. Hong, International Institute of Welding: IIW Doc. XIII-2036-04/XV-1173-04

[11] T. Gurney, Cumulative Damage of Welded Joints, Woodhead Publishing, Cambridge (2006). 